\title{
Employing Peer Support Workers in the Emergency Department to Help Care for Patients that Present After An Opioid-Related Overdose: Implementation Challenges and Recommendations From Key Stakeholders
}

Annette S. Crisanti ( $\square$ acrisanti@salud.unm.edu )

University of New Mexico

Jennifer Earheart

University of New Mexico

Research Article

Keywords: Peer support workers, Opioid Use Disorders, Emergency Departments

Posted Date: July 1st, 2021

DOI: https://doi.org/10.21203/rs.3.rs-653565/v1

License: (c) (i) This work is licensed under a Creative Commons Attribution 4.0 International License.

Read Full License 


\section{Abstract}

Background: The peer support model has been adapted in emergency departments (ED) throughout the country, specifically in response to increases in admissions resulting from opioid use disorder (OUD). The purpose of this study was to use community-engaged principles to identify implementation challenges and recommendations related to employing peer support workers (PSWs) in the ED to help care for patients that present after an opioid-related overdose.

Methods: A qualitative study was conducted to identify challenges to and recommendations for implementing the peer support model in the ED to help care for patients that present after an opioidrelated overdose. Nineteen stakeholders were interviewed, including directors/managers, nurses and PSWs. An inductive qualitative approach was used for the identification of themes from the interviews.

Results: Three themes surfaced among the implementation challenges, including system level challenges, hospital level challenges, and challenges specific to PSWs (referred to as individual level challenges). Recommendations to address the challenges are presented for successful integration of PSWs in ED settings to help care for patients that present after an opioid-related overdose.

Recommendations include, for example, clearly defined job descriptions and increasing understanding of what PSWs do among all hospital staff.

Conclusions: PSWs can play a role in the care for patients that present to the ED after an opioid-related overdose and in doing so help address the opioid crisis. Through the identification of challenges, awareness of strategies to address these challenges, and proper planning, hospitals can implement PSWs into the ED successfully. Planning should include thoughtful conversations between leadership, ED staff and, PSWs, as well as a commitment from leadership and hospital staff to recovery-oriented care. For the successful employment and integration of PSWs into ED settings to help care for patients that present after an opioid-related overdose, planning should focus on four key areas, including (1) hiring the right PSWs for the position, (2) education ED and hospital staff, (3) establishing protocols, and (4) training and supervision.

\section{Background}

An estimated 2 million people have an opioid use disorder (OUD). ${ }^{1}$ Between 2016 and 2017 the United States reported a $29.7 \%$ increase in the number of emergency department (ED) visits for opioid overdose. $^{2}$ The number of overdose deaths involving any opioid has more than doubled in the last 10 years (47,600 in 2017 compared to 18,515 in 2007). ${ }^{3}$ Risk of relapse is particularly high among those with OUD being discharged from prisons, inpatient units and detox centers. ${ }^{4}$ In New Mexico (NM) there was an $82 \%$ increase in the rate of opioid overdose ED visits from 2013-2015. ${ }^{5}$ In 2017 the rate of ED visits related to opioids was 52.3 per 100,000 . The most common drugs used in unintentional overdose are prescription opioids and heroin. ${ }^{5}$ 
With the rise of opioid overdose ED visits there is a need to make EDs "a critical entry point for primary and secondary prevention of opioid overdose".$^{6(\mathrm{p} .688)}$ When patients present at EDs with an opioid overdose or an opioid related event the goal is to get individuals stabilized and discharged as efficiently as possible. In some cases, a patient may be discharged with information on addiction resources, but referral to medication assisted treatment and follow-up are less common, especially in rural or remote areas. Consistent guidelines for post-care following an overdose are also limited. Vivolo-Kantor et al recommend EDs establish "post-overdose protocols that can help prevent subsequent overdose by providing naloxone and connecting patients with case management services or peer navigators to help link them into treatment and harm reduction services." ${ }^{2(p .284)}$ Studies have also shown that patients are more likely to engage in treatment and reduce their self-reported opioid use when medication assisted treatment is initiated in the ED. ${ }^{7}$

The peer support services model has shown to be effective in increasing social supports, patient engagement and well-being. ${ }^{8-9}$ Peer Support Workers (PSWs), also known as peer specialists, recovery coaches, or peer advocates, are individuals with lived experience with mental illness and/or substance use disorders who are successful in their recovery and deliver services in healthcare settings. Because of their shared experience with substance use, PSWs are able to better connect with people at the time of crisis.

Provision of peer support services is an established component of recovery-oriented care. Recently the peer support model has been adapted in EDs throughout the country, specifically in response to the opioid epidemic. ${ }^{10}$ Given the relative newness of the implementation of the peer support model in the ED, much of the literature to date focuses on feasibility, potential challenges, and guidelines for implementing peers in the ED. However, the limited but growing body of evidence thus far is positive. Outcome studies have shown that PSWs in the ED result in increased linkage to care; shorter days to initiation for substance use treatment; improved engagement with high risk populations and increased harm reduction education; and provision of naloxone. ${ }^{11-14}$

EDs are a unique healthcare environment. In the ED setting, staff are focused on achieving a high level of productivity within especially tight time constraints. Staff may face an unpredictable work environment, with long shifts, challenging patient loads and frequent exposure to potentially traumatic events. ${ }^{15}$ Staff workloads and emotional drain are frequently pointed as key stressors for staff in the ED. ${ }^{16}$ Within this work environment physicians and nurses experience a moderate to high level of burnout. ${ }^{17-18}$ PSWs working in this environment are exposed to the same challenges. With EDs being especially stressful environments with higher pressure, higher workloads and higher patient acuity compared to any other health care setting, PSWs working within EDs need to be especially flexible, responsive, agreeable, extroverted, and comfortable working with multi-disciplinary teams and within a stressful environment. In addition, PSWs working with EDs must be especially skilled at remaining calm amidst chaos and multitasking. While important for all PSWs, because of the nature of the work environment, those working 
within an ED setting must have superior coping skills and a high level of self-management, which involves taking an active role in one's recovery and wellness.

The purpose of this study was to use community-engaged principles to identify challenges to and recommendations for implementing the peer support model in the ED to help with care for patients with OUD. The goal of this work was to facilitate the implementation of the peer support model in EDs across the country to help address the opioid crisis.

\section{Methods}

In 2018, the NM Department of Health (NMDOH) received funding through the Centers for Disease Control (CDC) and Prevention's Emergency Response to address the opioid overdose epidemic. The CDC provides state, local, tribal, and territorial public health agencies money during identified public health emergencies through the Public Health Crisis Response grants. The CDC released the emergency Opioid Overdose Crisis Cooperative Agreement to advance the understanding of the opioid overdose epidemic and scale up prevention activities across all 50 States and Washington, D.C.. ${ }^{19}$ With its funds the NMDOH sought to enhance and expand an intervention aimed at addressing non-fatal opioid overdose admissions by incorporating one-on-one peer support services into EDs in four NM counties. To support the goal of successful implementation of their intervention, a qualitative study was conducted to identify challenges to and recommendations for implementing PSWs in the ED to help care for patients that present after an opioid-related overdose.

Study Participants

Nineteen stakeholders were interviewed, including ED and hospital directors/managers $(N=8)$, nurses $(\mathrm{N}=2), \mathrm{PSWs}(\mathrm{N}=4)$ and local and national experts who had expertise in the delivery of peer support services or with directly supervising or employing PSWs in ED settings $(\mathrm{N}=5)$. Except for the five "content" experts, study participants $(\mathrm{N}=14)$ worked in hospitals that, at the time of the interviews, incorporated peer support services in the ED $(\mathrm{N}=7)$, and hospitals that had not yet incorporated peer support services in the $E D(N=7)$ but were planning on doing so. Among this latter group, one of the participants worked in a rural community-based hospital. The other 13 participants $(\mathrm{N}=13)$ worked in hospitals located in urban areas. Stakeholders were identified using a combination of methods. First, with the assistance of the $\mathrm{NMDOH}$, stakeholders were identified from sites that were contracting with the $\mathrm{NMDOH}$ to incorporate peer support services into their ED. Key stakeholders were also identified using snowball sampling where interviewees were asked if they knew of someone else involved implementing PSWs in EDs that would be interested in being interviewed. ${ }^{20}$ Interviews were stopped once the interview content reached saturation. ${ }^{21}$

\section{Data Collection}

Interview guides were developed by the research team and were then shared with several stakeholders, including local PSWs, for cognitive testing of the questions (e.g., clarity and understanding) and to ensure 
the comprehensiveness of the questions. Stakeholders and PSWs who helped with refining the interview guides were excluded from the sampling pool. The authors conducted the interviews together and each took detailed notes. When possible, interviews were audio recorded so that narratives could be reviewed later for accuracy. Audio-recordings were unavailable for four interviews due to poor sound quality or interviews being conducted in an environment which prohibited the use of an audio-recorder. These interviews were still included in the analysis and stakeholders were recontacted if notes from the interviews needed to be clarified. Examples of open-ended questions included, for example: What current protocol is in place for patients presenting with an overdose? and Can you tell me more about the process or protocol for incorporating peers in the provision of services in the ED?

\section{Data Coding and Analysis}

An inductive qualitative approach was used for the identification of themes from the interviews. An inductive approach is commonly used in research when there is a large amount of textual data, there is little known about the subject, or when the researchers want to create potential explanations for the experiences that are present in the data. ${ }^{22}$ Each author independently read the transcriptions of the interviews thoroughly to identify key concepts. The major concepts were then compared and together the author coded the key concepts into themes. The themes were shared with interviewees to ensure that they summarized the key concepts accurately and thoroughly.

\section{Results}

Several challenges to incorporating PSWs within ED services were identified. Three themes surfaced among the implementation challenges, including system level challenges, hospital level challenges, and challenges specific to PSWs (referred to as individual level challenges).

While this conceptualization facilitates discussion, it is important to note that the challenges are not mutually exclusive. Table 1 provides examples of stakeholder's quotes for each theme. 
Table 1

Perceived Implementation Challenges and Examples of Related Participant Quotes

Themes Participant Quotes

\section{System Level Challenges}

PSW Workforce Shortages
"The primary issue we had with hiring for the position was that we had a ton of folks who applied for the position without meeting the position qualifications. We had a lot of people who were informal peer support workers but didn't have the certification or considered themselves peer support workers but didn't have the certification or had done a certification in another state and it was unclear whether or not they needed to be certified by the state of New Mexico or could have an equivalent."

Reimbursement for PSW Services
"We run a tight ship and there's not a lot of money to bring in other staff and so that's a real valid concern and l'd say that's true for all rural hospitals. We are staffed to a minimum core."

"The major challenge is going to just be finances in general. We're constantly having to keep an eye on our productivity. So adding another staff position without taking away a position that we already have filled

would be the huge challenge. Really being able to pitch that they need to add additional staff to our productivity."

\section{Hospital Level} Challenges 


\section{Themes}

Buy-in from

Providers and

Especially

Leadership

Mostly Resulting

from Not

Knowing who

PSWs are and

What They Can

Do

Logistics Related

to Integrating

PSWs into

Hospitals

\section{Participant Quotes}

"Selling our staff on the value and utilizing peers in the right ways would be the initial challenge. I think in the emergency department we have very strong personalities, it's very much 'I can do everything myself' so it would involve a little bit of a change in mindset."

"I could definitely see them helping out and filling in that role at times when there may not be an acute situation to assist with, just because with any of those patients I feel like if you have a past substance

abuse or psychiatric disorder I think you relate on a better level and are able to help those people calm and comfortable. So I could definitely see value in having them here."

"I think there's not a lot of understanding about what a PSW is. I don't know the exact role. I think there would be a lot of conversation about function between our counselor in the ED, social worker inpatient, and then how we would work as a whole team and what that would look like and what PSW could actually do."

"I don't know if anyone knows enough about it and maybe that's the challenge. We don't know enough about the use of PSWs. That I think in itself is a challenge. So it's like ok how does this mix with social work and what we have going on already. It's a knowledge deficit."

I was talking to staff and they didn't understand what the PSW is. They thought the SW in the term PSW was a social worker. And I said no it's not a social worker. So using that acronym was confusing."

"ED staff need to know what the peer support worker role is, why it's important, and how it benefits patient outcomes."

"The ED staff don't fully understand what a peer support worker can do. They quickly bought into the idea of peer support workers and believe it will be a good program but they don't fully understand what the role of the PSW is."

"We're constantly having to keep an eye on our productivity. So adding another staff position without taking away a position that we already have filled would be the huge challenge. Really being able to pitch that they need to add additional staff to our productivity."

"It's so ebb and flow. We don't know. We can't predict who's going to walk in the door. And so it doesn't make sense to a PSW here all of the time, but having an on call situation might be the right thing."

"It's important to have a clear process established on both sides so that everyone knows what is supposed to happen when a PSW is contacted. Otherwise, how are the ED staff going to know or remember in the chaos of everything going on to call for peer support?

"They worry that the peer would come in and in a small community like ours, is there going to be chitter chatter out in the community."
Concerns Related to

Professionalism 


\begin{tabular}{|ll|}
\hline Themes & Participant Quotes \\
\hline Supervision & $\begin{array}{l}\text { "It's so important to have good supervision. Having a plan for what to do if you } \\
\text { feel overwhelmed is important and the peer support worker needs to feel } \\
\text { comfortable with this person. Peers may be less likely to ask for help because } \\
\text { you wanted to be treated like everyone else, especially in this professional } \\
\text { position so you may not want to ask for help." }\end{array}$ \\
\hline "Current CPSW training does not include enough information on reporting or \\
documentation and documentation can be challenging for peers, especially in \\
the ED." \\
"Intensive training for peers working in the ED is important." \\
"Identifying the correct peer for the emergency room is the most difficult part of \\
this endeavor." \\
"The background of the PSW may make a difference for whether or not they \\
should be in the ED. If a PSW has a background in substance use, going to the \\
ED when someone has OD, can be really triggering".
\end{tabular}

\section{System Level Challenges}

Two themes were identified regarding system level challenges.

\section{PSW Workforce Shortages}

Stakeholders identified difficulties with finding PSWs who were interested in working within the ED and getting them to apply for ED based positions. One Administrator/Provider interviewed pointed to low compensation levels as a potential challenge to finding qualified applicants.

\section{Reimbursement for PSW Services}

- Another challenge at the system level related to the cost associated with funding the position. While there are currently many funding opportunities available for opioid related programs nationally, ED directors/managers were concerned with the costs of establishing and sustaining a PSW in the ED.

\section{Hospital Level Challenges}

Three themes were identified regarding hospital level challenges.

1. Buy-in from Providers and Especially Leadership Mostly Resulting from Not Knowing who PSWs are and What They Can Do

Stakeholders believed that for PSWs to be successful in their role in the ED (or other settings within the hospital), hospital providers and especially leadership must see the value PSWs can bring to the provision of services and the improvement of patient outcomes. In general, stakeholders indicated that leadership were in-support of peer services in the ED, as well as in other departments in the hospital. Though hospital leadership were in full support of peer support programs, several stakeholders 
mentioned that buy-in from other ED staff may present a challenge because of not knowing who PSWs are and what they do.

\section{Logistics related to integrating PSWs into the ED}

Leadership and hospital staff had questions about how best to integrate PSWs into the ED. More specifically, they had questions about: where the PSW should be located (including how to address lack of space at the hospital); whether the position should be part-time or full-time based on the caseload; what the hours of service should be; how the PSW will be notified when there is a patient that wants to be seen; what type of follow-up the PSW will provide and for how long; and what the role of the PSW will be and how will they be integrated into the provision of care.

\section{Concerns Related to Professionalism}

Stakeholders expressed concerns about whether PSWs understand the importance of HIPAA confidentiality (especially in rural communities with smaller populations) and representing the mission of the hospital. Stakeholders also expressed concerns about liability and the potential for PSWs to relapse.

\section{Individual (PSW) Level Challenges}

Three themes were identified regarding challenges at the PSW level (i.e., individual level challenges).

\section{Supervision}

Concerns around this theme focused on who would be the most appropriate supervisor (e.g., what should be the supervisor's background, training, and preparation for supervision) and whether there were any guidelines around supervision of a PSW.

\section{Training}

Stakeholders were concerned about the training for PSWs, whether PSWs would need training in a whole host of different topic areas and, if they were to be trained on the same topic as other ED staff, whether the training needed to be conducted separately. There were also concerns about whether the PSW training was comprehensive enough to provide a strong foundation for PSWs to succeed in what is seen as a non-traditional environment.

\section{Job Fit}

The ED can be a challenging workplace, with stressors unique to its environment and culture. All stakeholders interviewed agreed that job fit is of critical importance when hiring a PSW to work in the ED. 
Table 2

Checklist for the Successful Employment and Integration of PSWs into ED Settings to Help Care for Patients that Present after an Opioid-Related Overdose

\section{Hiring the Right Peer Support Worker (PSW)}

Emergency departments are stressful, high intensity environments. Hiring the right person for the position is important.
Develop a clearly defined job description so that PSWs applying for the position know what is expected.

Hire a PSW who is comfortable working with multidisciplinary teams, able to multi-task and remain calm amidst chaos, has superior coping skills and a high-level of self-management, which involves taking an active role in one's recovery and wellness.

Understand that some PSWs in the applicant pool may have a criminal background. Discussions with human resources around why this "lived experience" is important may be warranted.

\section{Educating ED \& Hospital Staff}

One of the biggest challenges to integrating PSWs in the ED is a lack of understanding of who PSWs are, what they do, their value, and what their role should be (referred to as PSW literacy).
Introduce PSWs to all ED staff including doctors, nurses, and pharmacists.

Explain the importance of the PSW role and how they will integrate with the ED team. Be sure to inform staff about how PSWs can help with challenging or frequent substance use patients.

Be specific about the role of the PSW including job expectations, requirements, and specific duties.

\section{Establishing Protocols}

To increase the likelihood of successful integration of PSWs in the ED, protocols must be established, reviewed, and revisited periodically.
Create a clear plan for how the PSW will respond. For example, will PSWs be contacted by ED staff or will they be stationed on-site.

Decide if the PSW will be tasked with following-up with patients, and if so for how long.

Determine what follow-up will look like (text message, phone call, or community visit).

\section{Training and Supervision}

Quality supervision and initial and ongoing training is essential for PSWs to thrive in their jobs.
Identify a supervisor and purpose and frequency of supervision.

Provide trainings on par with what other ED staff receive for PSWs to succeed in their job.

\section{Discussion}

Key stakeholders identified several challenges to implementing PSWs in the ED to help care for patients that present after an opioid-related overdose. Several of the challenges that were identified by 
stakeholders are consistent with those identified in the literature. ${ }^{10}$ Conceptually, the challenges spanned the hospital, system and individual levels but noteworthy is that the challenges are not mutually exclusive. Challenges at the hospital level include difficulty in recruiting qualified applicants and payment for PSW services. Challenges at the hospital level include (i) buy-in from providers, especially leadership, mostly resulting from not knowing who PSWs are and what they can do; (ii) logistics related to integrating PSWs into the ED, and (iii) concerns related to professionalism. Challenges at the individual level include (i) supervision, (ii) training, and (iii) job fit. Recommendations to address the challenges identified at each level are discussed below.

\section{Recommendations to Address Challenges at the System Level}

PSW Workforce Shortages (Especially as it relates to hiring within the ED): Peer certification and re-certification courses may provide an opportunity to introduce PSWs to the ED with the goal of increasing the number of PSWs interested in working within this setting. In NM, peers are required to complete 40 experiential hours, which can include volunteer or work time at an approved organization, before they can obtain a board-certified PSW Certification (CPSW). This requirement could provide an opportunity for PSWs to job shadow other providers (e.g., counselors) in the ED to get familiar and comfortable with the setting. In larger urban counties that are more likely to have access to an applicant pool of PSWs, it may be beneficial for hospitals to host or join a planned job fair to increase awareness of current employment opportunities. Hospitals are also encouraged to work with state mental health agencies who can disseminate information on job opportunities to their listserv. Finally, given that PSWs are often required to complete continuing education, hospitals may want to partner with certification boards to develop a course on "The Role of PSWs in the Emergency Department" to provide an overview of the unique characteristics of the setting and expected job-related duties. This course would not only increase awareness of PSW employment opportunities in a non-traditional setting but would allow PSWs a platform to gather information and ask questions to make an informed decision regarding job fit.

Stakeholders attributed the difficulty in getting PSWs to apply for positions in the ED to low compensation levels. A survey of PSWs in Georgia found that PSWs were likely to be unemployed and those that were employed were in positions with limited benefits and low income, $\$ 10,000-\$ 20,000.23$ Competitive salaries along with comprehensive benefit packages could increase the number of PSWs applying for positions in the ED.

Although not identified as a challenge in this qualitative study, hospital leadership should be aware of human resource policies for criminal background checks that may impede some qualified candidates from applying for positions in the hospital. For various reasons, individuals with mental illness and/or substance use disorders are at increased risk for involvement in the criminal justice system. ${ }^{24}$ As a result, some PSWs may have a criminal justice background which creates hiring challenges for hospitals that are restricted from employing individuals with a criminal background. Leadership will need to work with human resources to help them understand that it is "lived experience" that makes PSWs valuable and effective in what they do. As described by Richardson \& Rosenberg, it is important that PSWs "are seen as 
assets in peer support programs, because the specificity of their lived experience is useful in engagement and relationship building." 10(p.6) To counter this particular challenge some hospitals chose to partner with treatment providers based in the community whose hiring practices do not limit applicants.

Reimbursement for PSW Services: With respect to cost, hospitals should look to other funding sources such as Medicaid 1115 waivers and Medicaid State Plan Amendments. ${ }^{10}$ In NM, the Medical Assistance Division recognizes CPSWs who hold a certification from the NM credentialing board as members of the behavioral health workforce who can deliver reimbursable services. The State of NM Medical Assistance Program Manual Supplement and associated policy and billing manual provide detailed information about Medicaid reimbursable services that can be delivered by CPSWs. The majority of these services are delivered in outpatient and community-based settings. Only one identified Medicaid service (SBIRT) is currently reimbursable in emergency department settings.

Although funding PSW positions may require the development of creative reimbursement processes, there could be potential cost savings for hospitals who implement peer services. Due to the nature of their illness, patients with OUD may rely on of emergency services for their healthcare more than others. Often these patients are referred to as high utilizers. A study conducted in Delaware found that patients who engaged in a brief intervention (which included Motivational Interviewing) led by a PSW had improved healthcare utilization and could be correlated to cost savings. In one cohort, patients who were connected to substance use treatment through the PSW had a $58 \%$ decrease in inpatient medical admissions $(\$ 68,422) ; 13 \%$ decrease in ED visits $(\$ 3,308) ; 32 \%$ decrease in behavioral health inpatient admissions $(\$ 18,119)$; and a 32\% decrease in outpatient admissions $(\$ 963)$. Among this cohort of 25 this represents a $\$ 88,886$ difference in healthcare costs. ${ }^{25}$

\section{Recommendations to Address Challenges at the Hospital Level}

Buy-in from Providers and Especially Leadership Mostly Resulting from Not Knowing who PSWs are and What They Can Do: For ED directors/managers and ED staff to buy into the idea of employing and working side by side with PSWs it is critical that they have a full understanding of what PSWs do and how best to integrate them within the ED in response to OUD. ${ }^{26}$ The successful integration of PSWs in EDs requires leadership and other stakeholders to have "PSW literacy", defined as a full understanding of who PSWs are, what they do (e.g., what is their role), what is their professional and required training, and what are the benefits of incorporating PSWs in the provision of care. PSW literacy can be increased by providing literature to leadership and providers on PSWs and their effectiveness as well as in-service trainings and orientations for new staff. It is important for all hospital staff to have an understanding of the role and effectiveness of PSWs before they are incorporated into an existing team. In a study of the integration of PSWs in mental health agencies in New York researchers found that a source of conflict arose when staff were not prepared for the inclusion of peer support workers. ${ }^{27}$ The greater support from all levels of providers the more successful the PSW will be. Several of the PSWs interviewed mentioned the importance of building relationships with ED staff and the impact those relationships had on their engagement with patients. One PSW interviewed suggested a possible solution to the buy-in challenge is 
to simply have the PSW round with the hospital team or participate in team meetings. This approach is also recommended by Richardson and Rosenberg (n.d.), as well as Gates and Akabas (2007) who further suggest that strategies to build relationships between PSWs and other staff focus on effective communication methods of information sharing related to patient cases and opportunities to increase mutual understanding and support. ${ }^{10,27}$

Logistics related to integrating PSWs into the ED: While there have been various ways in which hospitals have implemented PSWs into the system of care at the national level, there are two overarching models. The first and most common model is that hospitals develop a memorandum of understanding with a recovery community organization, or RCO. ${ }^{10}$ The second model is that hospitals hire the peers directly. There are several advantages to hospitals partnering with recovery organizations in the community. First, community organizations have a deeper understanding and knowledge of PSWs and their role in recovery. Second, PSWs have routine access to supervision at recovery community organizations. Third, it helps hospital staff understand the role of PSWs before committing to their own program. Fourth, if an individual is not ready for services at the time of an overdose, but decides they would like services later on, they will have a connection to the resource community organization. Fifth, partnering with community organizations sometimes provides a "buffer zone" on the ability to hire PSWs who may have a criminal background. The first step in the integration of PSWs in the ED is deciding on the model that is most appropriate, which will vary from hospital to hospital. In deciding which model is the best fit, administrators need to consider logistics such as need, space, patient-flow (including triage), and cost. An evaluation of State Targeted Response funded peer services in the ED also found concerns related to integrating PSWs in the EDs. In New Jersey, Nevada, and Indiana administrators were concerned with where peers would be based (physically and administratively), how the peers would be notified of an eligible patient, how to make patients aware of peer services, and what post-treatment strategies should be used. There were a few commonalities among the 22 programs interviewed, including that there were no programs in which peers were administratively housed in the ED and in most hospitals, ED staff are the first to introduce patients to peer services, even if peers had access to ED admissions and could identify patients beforehand. ${ }^{28}$

Concerns Related to Professionalism: Most of these concerns could be alleviated by increasing leaderships' awareness about what is covered in the PSW training. For example, in most certification programs an entire session is dedicated to HIPAA and related confidentiality regulations. In NM one of the CPSW training sessions focuses on the five elements of professionalism, including dependability, dress, demeanor, diplomacy and discretion. As a result of the CPSW training in NM, PSWs are expected to know and exercise basic skills and competencies, including cultural competence, trauma-informed and sharedawareness and continuous critical learning. PSWs should also be aware of hospital policies regarding violations of confidentiality and other issues that may increase liability (e.g., development of a dual relationship). Such policies, and consequences for violating them, should be made clear to the PSW upon hiring and any issues should be addressed quickly and regularly during supervision. With respect to concerns about relapse, Chinman et al stated that there is: "no evidence that the demands of work 
exacerbate health conditions or lead to relapses among peer specialists. In fact, meaningful, competitive work may serve to enhance recovery. Research indicates that employment is linked to beneficial effects on clinical and social functioning." $29(\mathrm{p} .21)$

Furthermore, according to a technical guide for clinical staff on how best to integrate consumer providers into staff culture, "the persistent misconception that consumer providers will inevitably relapse should be addressed and dispelled." 30 (p.17) While relapse is not common, it may happen. Because of this, PSWs should be encouraged to share with their supervisor when their symptoms are becoming symptomatic and supervisors should be encouraged to tactfully point out behaviors that a PSW may be exhibiting that may be of concern. PSWs should be handled like all other employees who have an illness that may interfere with job performance. Similar to other employees, PSWs need to be encouraged to take sick time, including wellness days if available, when needed, and they need to know that they can return to their jobs when they are well enough to perform work related duties. Quality supervision is essential for PSWs to thrive in their jobs and to reduce the likelihood of the professional issues (e.g., violations of confidentiality).

\section{Recommendations to Address Challenges at the Individual Level}

Supervision: In 2014, the Pillars of Peer Support Services Summit developed Pillars of Peer Support Supervision to guide the evolving growth of peer support services and the workforce that provides them. ${ }^{31}$ Based on input from national experts, supervision of peer specialists is most effective when supervisors: 1. Are Trained in Quality Supervisory Skills; 2. Understand and Support the Role of the Peer Specialist, 3. Understand and Promote Recovery in their Supervisory Roles, 4. Advocate for the Peer Specialist and Peer Specialist Services Across the Organization and in the Community, and 5. Promote the Professional Growth of the Peer.

Training: PSWs who work in the ED may indeed need additional training on topics related to the environment. Information on other people's roles in the ED, policies and procedures, values around safety and clinical expertise were also identified by one of the experts. Professional development opportunities are important for PSWs. A study on the experiences of PSWs found that $89 \%$ believed that additional training in special topics would improve their professional experience. ${ }^{23}$ Training could include but should not be limited to: Motivational Interviewing; Safety policies and procedures in the ED; Working in stressful environments; How to be part of an ED team; and The importance of self-care. Also, training should include policy and procedures as well as detailed information about job expectations, requirements, and specific duties. With respect to the issue of whether PSWs can benefit from the same training provided to other hospital staff (referred to as a one-size fits all training approach versus different trainings for learners that vary by educational background), there is no evidence to suggest the need for different versions of the same training. In a study on the effectiveness of a one-day training on Seeking Safety (an evidence-based trauma specific treatment), for PSWs and behavioral health practitioners, Crisanti et al. found that the former group benefited from the training to the same extent as 
the latter group. Furthermore, post-training feedback on satisfaction with the training and perceived comfort level in implementing the evidence-based practice did not differ among participants. ${ }^{32}$

Job Fit: Job fit is a concept that underscores the importance of matching an employee's strengths, needs and experience to requirements of a particular job and work environment. ${ }^{33} \mathrm{Job}$ fit is important because it results in higher levels of satisfaction and mental and physical well-being. ${ }^{34}$ PSWs working within EDs need to be especially flexible, responsive, agreeable, extroverted, and comfortable working with multidisciplinary teams and within a stressful environment. PSWs working within EDs must be skilled at remaining calm amidst chaos and multi-tasking. While important for all PSWs, because of the nature of the work environment, those working with an ED setting must have superior coping skills and a high level of self-management, which involves taking an active role in one's recovery and wellness. PSWs hired to work in the ED should be well established in their recovery and have a strong sense of resiliency. Self-care is especially important. In interviews with PSWs in Canada participants noted there was a disconnect between the training they received and the stressful nature of their work ${ }^{26}$ Although they may be more challenging to provide for PSWs working in rural communities, peers should have opportunities for networking and professional growth. In addition to focusing on job fit and self-care it is important for hospitals to develop clear, well-defined job descriptions for the hiring process. Lack of role clarity is mentioned in the literature and was discussed in our interviews. ${ }^{27,35 ~ " P o o r l y ~ d e f i n e d ~ j o b ~ d e s c r i p t i o n s ~}$ make it difficult for peer support workers to be successful, and hinder their integration into multidisciplinary work teams"35(p.2)

\section{Strengths and Limitations}

Qualitative interviews were conducted with administrators, providers, PSWs as well as experts in peer delivered services thereby providing diverse insight to challenges associated with employing PSWs in the ED. However, only one of our stakeholders worked in a hospital located in a rural community, and because rural hospitals in NM had yet to employ PSWs at the time this study was conducted, the PSWs that were interviewed were all employed within urban hospital settings. While it is likely that rural hospitals will experience many of the challenges identified in this qualitative study, there may be additional challenges to implementing the peer recovery model in EDs in hospitals in rural settings. Certainly, leadership and staff in hospitals located in rural communities may need to be more innovative in the ways in which they decide to incorporate PSWs into their system of care to address the OUD problem. Finally, audio-recordings were unavailable for four interviews due to poor sound quality or interviews being conducted in an environment which prohibited the use of an audio-recorder.

\section{Conclusions}

PSWs can play a role in the care for patients that present to the ED after an opioid-related overdose and in doing so help address the opioid crisis. Through the identification of challenges, awareness of strategies to address these challenges, and proper planning, hospitals can implement PSWs into the ED successfully. Planning should include thoughtful conversations between leadership, ED staff and, PSWs, 
as well as a commitment from leadership and hospital staff to recovery-oriented care. For the successful employment and integration of PSWs into ED settings to help care for patients that present after an opioid-related overdose, planning should focus on four key areas, including (1) hiring the right PSWs for the position, (2) education ED and hospital staff, (3) establishing protocols, and (4) training and supervision. Table 2 includes a checklist of items within each of these four areas that may be helpful to consider to increase the likelihood of successful employment of PSWs in the ED and their retention in a challenging work setting.

\section{Abbreviations}

- $C D C$ - Centers for Disease Control

- CPSW- Certified Peer Support Worker

- ED - Emergency Department

- NM - New Mexico

- NMDOH - New Mexico Department of Health

- OUD - Opioid Use Disorder

- PSWs - Peer Support Workers

\section{Declarations}

Ethics approval and consent to participate: This study was approved by the author's affiliated University's Human Research Protections Office, Institutional Review Board (IRB, ID\# 20-244). The IRB determined that informed consent was not required as interviews were de-identified. Methods were carried out in accordance with national, institutional, and international guidelines and regulations.

Consent for publication: Not applicable.

Availability of data and materials: Data can be accessed by contacting the first author.

Competing Interests: The authors have no relevant financial or non-financial conflicts to disclose.

Funding: The study was funded through the New Mexico Department of Health (NMDOH), which received funding from CDC Opioid Overdose Crisis Cooperative ("Public Health Crisis Notice of Funding Opportunity | CDC," 2018).

Authors Contributions: Authors certify (a) that they accept responsibility for the conduct of the study and for the analysis and interpretation of the data, (b) that they helped write the manuscript and agree with the decisions about it, (c) that they meet the definition of an author as stated by the International Committee of Medical Journal Editors, and (d) that they have read and approved the final manuscript.

Acknowledgements: Not applicable 


\section{References}

1. Substance Abuse and Mental Health Services Administration. Key Substance Use and Mental Health Indicators in the United States: Results from the 2018 National Survey on Drug Use and Health. NSDUH Series H-54, HHS Publication No. PEP19-5068, Rockville, MD: Center for Behavioral Health Statistics and Quality, 2019.

2. Vivolo-Kantor AM, Seth P, Gladden M., et al. Vital Signs: Trends in Emergency Department Visits for Suspected Opioid Overdoses - United States, July 2016-September 2017. US Department of Health and Human Services, Centers for Disease Control and Prevention. Morbidity and Mortality Weekly Report. 2018;67(9):279-285. doi:10.15585/mmwr.mm6709e1

3. Centers for Disease Control and Prevention, National Center for Health Statistics. Multiple Cause of Death 1999-2019 on CDC WONDER Online Database. National Drug Overdose Deaths Involving Any Opioid.

4. Nunes EV, Gordon M, Friedmann PD, et al. Relapse to Opioid Use Disorder After Inpatient Treatment: Protective Effect of Injection Naltrexone. Journal of Substance Abuse Treatment. 2018;85:49-55. doi:10.1016/j.jsat.2017.04.016

5. New Mexico Department of Health. New Mexico Substance Use Epidemiology Profile, 2018.

6. Houry DE, Haegerich TM, Vivolo-Kantor A. Opportunities for Prevention and Intervention of Opioid Overdose in the Emergency Department. Annals of Emergency Medicine. 2018;71(6):688-690. doi:10.1016/j.annemergmed.2018.01.052

7. D'Onofrio G, O'Connor PG, Pantalon MV, et al. Emergency Department-Initiated Buprenorphine/Naloxone Treatment for Opioid Dependence: A Randomized Clinical Trial. JAMA. 2015;313(16):1636-1644. doi:10.1001/jama.2015.3474

8. Bassuk EL, Hanson J, Greene RN, et al. Peer-Delivered Recovery Support Services for Addictions in the United States: A Systematic Review. Journal of Substance Abuse Treatment. 2016;63:1-9. doi:10.1016/j.jsat.2016.01.003

9. Reif S, Braude L, Lyman DR, et al. Peer Recovery Support for Individuals With Substance Use Disorders: Assessing the Evidence. Psychiatric Services. 2014;65(7):853-861. doi:10.1176/appi.ps.201400047

10. Richardson J, Rosenberg L. Peer Support Workers in Emergency Departments: Engaging Individuals Surviving Opioid Overdoses - A Qualitative Assessment. National Council for Behavioral Health.

11. Carey $\mathrm{CW}$, Jones $\mathrm{R}$, Yarborough $\mathrm{H}$, et al. Peer-to-Peer Addiction Counseling Initiated in the Emergency Department Leads to High Initial Opioid Recovery Rates. Annals of Emergency Medicine. 2018;72(4):S143-S144. doi:10.1016/j.annemergmed.2018.08.371

12. Samuels EA, Bernstein SL, Marshall BDL, et al. Peer Navigation and Take-home Naloxone for Opioid Overdose Emergency Department Patients: Preliminary Patient Outcomes. Journal of Substance Abuse Treatment. 2018;94:29-34. doi:10.1016/j.jsat.2018.07.013 
13. Waye KM, Goyer J, Dettor D, et al. Implementing Peer Recovery Services for Overdose Prevention in Rhode Island: An Examination of Two Outreach-based Approaches. Addictive Behaviors. 2019;89:85-91. doi:10.1016/j.addbeh.2018.09.027

14. Samuels EA, Baird J, Yang ES, et al. Adoption and Utilization of an Emergency Department Naloxone Distribution and Peer Recovery Coach Consultation Program. Society for Academic Emergency Medicine. 2018;0(0). doi:10.1111/acem.13545

15. Schneider A, Weigl M. Associations Between Psychosocial Work Factors and Provider Mental Wellbeing in Emergency Departments: A Systematic Review. PLOS ONE. 2018;13(6):e0197375. doi:10.1371/journal.pone.0197375

16. Johnston A, Abraham L, Greenslade J, et al. Review Article: Staff Perception of the Emergency Department Working Environment: Integrative Review of the Literature. Emergency Medicine Australasia. 2016;28(1):7-26. doi:10.1111/1742-6723.12522

17. Bragard I, Dupuis G, Fleet R. Quality of Work Life, Burnout, and Stress in Emergency Department Physicians: A Qualitative Review. European Journal of Emergency Medicine. 2015;22(4):227-234. doi:10.1097/MEJ.0000000000000194

18. Hunsaker S, Chen H-C, Maughan D, et al. Factors That Influence the Development of Compassion Fatigue, Burnout, and Compassion Satisfaction in Emergency Department Nurses. Journal of Nursing Scholarship. 2015;47(2):186-194. doi:10.1111/jnu.12122

19. Centers for Disease Control and Prevention. Public Health Crisis Notice of Funding Opportunity. Available online at https://www.cdc.gov/cpr/readiness/funding-crisis.htm. Accessed October 25, 2019.

20. Biernacki P, Waldorf D. Snowball Sampling: Problems and Techniques of Chain Referral Sampling. Sociological Methods and Research. 1981;10(2):141-163.

21. Saunders B, Sim J, Kingstone T, et al. Saturation in Qualitative Research: Exploring its Conceptualization and Operationalization. Quality and Quantity. 2018;52(4):1893-1907. doi:10.1007/s11135-017-0574-8

22. Thomas DR. A General Inductive Approach for Analyzing Qualitative Evaluation Data. American Journal of Evaluation. 2006;27(2):237-246. doi:10.1177/1098214005283748

23. Ahmed A, Hunter K, Mabe A, et al. The Professional Experiences of Peer Specialists in the Georgia Mental Health Consumer Network. Community Mental Health Journal. 2015;51:424-436.

24. Prince J, Wald C. Risk of Criminal Justice System Involvement Among People with Co-occurring Severe Mental Illness and Substance Use Disorder. International Journal of Law and Psychiatry. 2018;58:1-8. doi:10.1016/j.ijlp.2018.02.002

25. Pecoraro A, Horton T, Ewen E, et al. Early Data from Project Engage: A Program to Identify and Transition Medically Hospitalized Patients into Addictions Treatment. Addiction Science and Clinical Practice. 2012;7(1):20. doi:10.1186/1940-0640-7-20

26. Gruhl KLR, LaCarte S, Calixte S. Authentic Peer Support Work: Challenges and Opportunities for an Evolving Occupation. Journal of Mental Health. 2016;25(1):78-86. 
doi:10.3109/09638237.2015.1057322

27. Gates LB, Akabas SH. Developing Strategies to Integrate Peer Providers into the Staff of Mental Health Agencies. Administration and Policy in Mental Health Services Research. 2007;34(3):293306. doi:10.1007/s10488-006-0109-4

28. McGuire AB, Powell KG, Treitler PC, et al. Emergency Department-Based Peer Support for Opioid Use Disorders: Emergent Functions and Forms. Journal of Substance Abuse Treatment. 2020;108:82-87. doi:10.1016/j.jsat.2019.06.013

29. Morris CW, Banning LB, Mumby SJ, Morris CD. Dimensions: Peer Support Program Toolkit. University of Colorado Anschultz Medical Campus, School of Medicine, Behavioral Health and Wellness Program. 2015:1-51. https://www.bhwellness.org/toolkits/Peer-Support-Program-Toolkit.pdf.

30. Chinman M, Hamilton A, Butler B, et al. Mental Health Consumer Providers: A Guide for Clinical Staff. RAND Corporation. 2008.

31. Daniels AS, Tunner TP, Powell I, et al. Pillars of Peer Support Services Summit Six: Peer Specialist Supervision. Annual Summit. Atlanta, Georgia, October 2014.

32. Crisanti AS, Murray-Krezan C, Karlin LS, et al. Evaluation of an Evidence-based Practice Training for Peer Support Workers in Behavioral Health Care. Cogent Psychology. 2016;3(1):1212453. doi:10.1080/23311908.2016.1212453

33. Heathfield, S. Assess Job Fit When You Select Your Employees. The Balance Careers. Published July 27, 2019. Available online at https://www.thebalancecareers.com/assess-job-fit-when-you-selectemployees-1918165. Accessed November 25, 2019.

34. Tinsley $\mathrm{H}$. The Congruence Myth: An Analysis of the Efficacy of the Person-Environment Fit Model. Journal of Vocational Behavior. 2000;56:147-179. doi:10.1006/jvbe.1999.1727

35. Jacobson N, Trojanowski L, Dewa CS. What Do Peer Support Workers Do? A Job Description. BMC Health Services Research. 2012;12(1):205. doi:10.1186/1472-6963-12-205

\section{Figures}




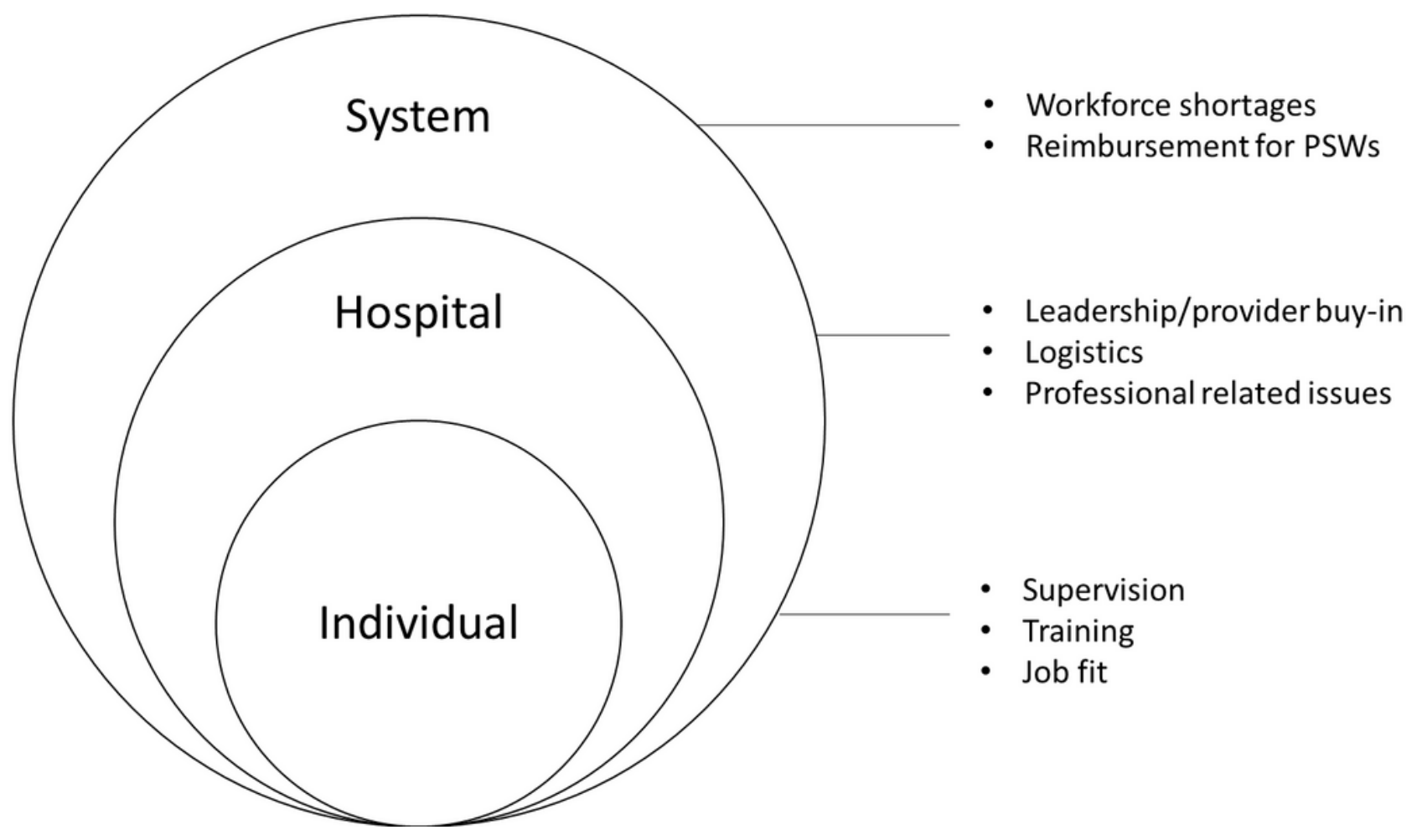

\section{Figure 1}

Implementation Challenges Identified by Key Stakeholders in Employing Peer Support Workers in the Emergency Department to Help Care for Patients that Present after an Opioid-Related Overdose

\section{Supplementary Files}

This is a list of supplementary files associated with this preprint. Click to download.

- SupplementInterviewGuides.docx 